\title{
Effect of Organic Nutrients and Bio-mulches on Growth, Physiology and Yield of Okra (Abelmoschus esculentus L. Moench)
}

\author{
Subhalaxmi Mishra ${ }^{1 *}$, Satyamaya Satapathy ${ }^{2}$, Ranjit Chatterjee ${ }^{3}$ and Koushik Saha ${ }^{4}$ \\ ${ }^{1}$ Department of Vegetable Science, OUAT, Bhubaneswar, 751003, India \\ ${ }^{2}$ Agronomy, KVK, OUAT, India \\ ${ }^{3}$ Department of Vegetable and Spice Crops, UBKV, 736165, India \\ ${ }^{4}$ Division of Vegetable Crops, IIHR, Bengaluru, 560089, India
}

*Corresponding author

\begin{tabular}{|l}
\hline K e y w o r d s \\
Organic nutrients, \\
Bio-mulch, Bio- \\
fertilizers, Bio- \\
stimulants
\end{tabular}

\section{Introduction}

Okra, popularly known as Bhindi is one of the leading vegetable in the country. India is the leading producer of okra. It is rich source of protein vitamins and minerals. Medicinal properties of okra include control of goitre
(Yawalkar, 1965), useful against genitorurinary disorders, spermatorrhoea and chronic dysentery (Nandakarni, 1927) and relief from haemorrhoids (Adams, 1975) etc. Okra mucilage has potential for use as food, nonfood products, and medicine. Dried stems and roots of okra are used for cleaning sugarcane 
juice from which molasses is prepared. The dry seeds are rich source of oil (18-20\%) and crude protein (20-23\%). It has occupied a prominent position among the export oriented vegetables in India because of high nutritive value, palatability and good post- harvest life. It has an enormous potential as one of the foreign exchange earner crops and accounts for $70 \%$ of the export of fresh vegetables (Dhankhar and Mishra, 2005). At present, it is being exported to the neighboring countries in the Gulf and South- East Asia, particularly Singapore, Mauritius, Malaysia, Sri Lanka and Bangladesh.

Being a heavy manuring crop it requires optimum nutrient for its growth and development. But indiscriminate uses of chemical fertilizers adversely affect the plant growth, disease pest infestation and residual toxicity of fruit and in long run decreases the soil fertility. Application of nutrient through the organic source is the only way to overcome all these obstacles. Another barrier in the conventional crop production is the growing public concern about the ill effect of chemical fertilizers.

The new emerging organic supplements like seaweed extract and humic acid along with farm yard manure and vermi compost modify the soil physical, chemical and biological properties and facilitate a sustainable crop growth. These nutrient source promote plant physiology and mechanism (Aydin et al., 2012), disease resistance and plant hormone activities (Zodape et al., 2011).

Maintenance of root micro-climate during the plant growth is very essential. Covering of the soil surface is known as mulching. This practice maintains soil temperature, inhibits weed growth, retains optimum moisture in soil and favors quality and early harvest. In general polythene sheets of various micron sizes are used as mulch material. But it has several disadvantages like difficulty of removal, cost of disposal, increased soil erosion and increased agricultural chemical runoff (Lamont, 1993; Hochmuth, 1998; Brown and Channell- Butcher, 2001; Rice et al., 2001). In a fully organic crop production system this can be replaced by organic mulches such as straw, dry leaves etc. which are easily available and degradable. However there is lack of application of locally available mulch materials like dry water hyacinth, dry weeds and legume plant residue which can be fully exploited.

Keeping all these aspects on view a research work was framed to study the combined effect of organic nutrient and organic mulch on okra growth, yield and physiology.

\section{Materials and Methods}

The field experiments were conducted at Instructional farm of Department of Vegetable Science, UBKV, Pundibari, Coochbehar, West Bengal, India. The place is situated at Terai Zone of West Bengal. Experiment was laid out in Randomized block Design with three replications. There were two factors of the experiment. Factor 'N' Organic Nutrient Sources and Factor M - Bio mulches, each factor having four levels each. The levels under organic nutrient sources $\mathrm{N}$ were $\mathrm{N}_{1}-$ Farmyard manure $(25$ t/ha $)+$ Azophos biofertiliser + Humic Acid; $\mathrm{N}_{2}$ - Farmyard manure (25 t/ha) + Azophos biofertiliser + Seaweed extract; $\mathrm{N}_{3}$-Vermicompost (5 t/ha) + Azophos biofertiliser + Humic acid; $\mathrm{N}_{4}$ Vermicompost (5 t/ha) + Azophos biofertiliser +Seaweed extract and levels of bio-mulches namely $\mathrm{M}_{1}$ - Rice Straw; $\mathrm{M}_{2-}$ Dry water hyacinth (Eichhornia crassipes); $\mathrm{M}_{3^{-}}$Dry local weed (Polygonum persicaria); $\mathrm{M}_{4}$ - Dry vegetable legume plant residues which comprises of 16 treatment and combination in total. Okra (cv. Arka Anamika) seeds (@20 $\mathrm{Kg} / \mathrm{ha}$ ) were sown for both the year with spacing of $45 \mathrm{cmX} 45 \mathrm{~cm}$. To prepare enriched organic manure, the Azophos biofertiliser 
(Azotobacter + Phosphate Solubilizing Bacteria) was mixed with vermicompost and farmyard manure and kept in moist and dark condition for 15 days before field application. Humic acid and seaweed extract (Ascophyllum nodosum) were sprayed on standing crop at 30, 40 and 50 days after sowing (DAS) at the rate of $2 \mathrm{ml} / \mathrm{l}$ (Kumari et al., 2011). Mulching (10 t/ha) was done just after thinning of seedlings. All the standard cultural practices were adopted during the crop growth period. Harvesting of the pods was done after 6 days of anthesis and was continued up to $7^{\text {th }}$ picking depending upon marketable stage. Random samples were collected from each treatment for the assessment of several parameters like growth, physiology and yield. Two years data collected were pooled and statistically analyzed by using INDOSTAT statistical package (version 7.00, Hyderabad, India).

\section{Results and Discussion}

\section{Effect of organic nutrient sources}

The data relating to growth parameter of okra has been presented in the table 1 and 2. The data depicted that among all the nutrient sources, biofertiliser enriched vermicompost with foliar application of seaweed extract showed highest plant height at 60 days after sowing in both the year with pooled value $65.85 \mathrm{~cm}$.

Similarly for the same treatment $\mathrm{N}_{4}$ maximum stem girth at the time of last harvest was observed in both years with pooled value $20.05 \mathrm{~mm}$. There was no significance of nutrient sources on node no. to first flowering and number of branches per plant.

However the same treatment $\mathrm{N}_{4}$ exhibited minimum days to $50 \%$ flowering (48.18 days in pooled analysis) and days to first harvest, that is 54.19 days. In case of days to last harvest $\mathrm{N}_{4}$ showed maximum days 86.24 in pooled followed by $\mathrm{N}_{2}$ (bio fertiliser enriched farm yard manure with foliar spray of seaweed extract).

The nutrient source biofertiliser enriched vermi compost with 3 time foliar spray of seaweed extract emerged better in relation to the physiological attributes of the okra plant. Leaf chlorophyll content was more at 60 DAS for the treatment $\mathrm{N}_{4} 39.88$ SPAD value in pooled. Highest crop growth rate at 45-60 DAS (4.59 $\mathrm{gram} / \mathrm{m}^{2} /$ day in pooled) was recorded by the same treatment followed by $\mathrm{N}_{2} \quad$ (4.26 gram $/ \mathrm{m}^{2} /$ day in pooled). Subsequently greater accumulation of dry matter $(23.85 \%)$ was observed at 60 DAS for the same treatment.

The pooled data revealed that the yield attributing character varied significantly with the different organic nutrient sources. The okra plant, where biofertiliser enriched vermicompost with three time foliar spray of seaweed extract was applied resulted maximum no. of fruit per plant (19.94), yield per plant $(322.15 \mathrm{~g})$ and highest total yield per hectare (14.39 t/ha).

Vermicompost has high mineralization rate than farmyard manure and assured slow releases of major plant nutrients like $\mathrm{N}, \mathrm{P}$ and $\mathrm{K}$ and when combined with bio-stimulant like seaweed extract promoted the high plant growth due to certain hormonal content like auxin, gibberellic acid and cytokinin. All these might have induced the plant metabolism and exhibited better plant vigor and subsequently resulted increase yield in okra.

Zodape et al., (2011) pointed that application of 5\% seaweed extract as foliar spray and Azotobacter biofertiliser enriched vermicompost (Gopinathan and Prakash, 2004) indicated greater plant height as well as yield in tomato. 
Table.1 Effect of organic nutrients and bio-mulches on growth parameters of okra

\begin{tabular}{|c|c|c|c|c|c|c|c|c|c|c|c|c|}
\hline Treatment* & $\begin{array}{l}\text { Plant height at } \\
60 \text { Days after } \\
\text { sowing }(\mathrm{cm})\end{array}$ & & & $\begin{array}{l}\text { Stem girth at } \\
\text { the time of last } \\
\text { harvest (mm) }\end{array}$ & & & $\begin{array}{l}\text { No. of } \\
\text { branches } \\
\text { per plant }\end{array}$ & & & $\begin{array}{l}\text { Node number } \\
\text { to first } \\
\text { flowering }\end{array}$ & & \\
\hline & 2017 & 2018 & pooled & 2017 & 2018 & pooled & 2017 & 2018 & pooled & 2017 & 2018 & Pooled \\
\hline \multicolumn{13}{|c|}{ Nutrient sources } \\
\hline $\mathbf{N}_{1}$ & 63.23 & 64.37 & 63.80 & 18.57 & 18.63 & 18.60 & 2.75 & 2.50 & 2.63 & 4.00 & 3.99 & 4.00 \\
\hline $\mathbf{N}_{2}$ & 64.43 & 64.25 & 64.34 & 17.95 & 18.92 & 18.44 & 2.97 & 2.81 & 2.89 & 3.65 & 3.92 & 3.79 \\
\hline $\mathbf{N}_{3}$ & 65.27 & 65.17 & 65.22 & 17.98 & 17.70 & 17.84 & 2.69 & 2.70 & 2.70 & 4.21 & 3.91 & 4.06 \\
\hline $\mathbf{N}_{4}$ & 65.58 & 66.12 & 65.85 & 19.73 & 20.37 & 20.05 & 2.86 & 2.84 & 2.85 & 3.56 & 3.53 & 3.54 \\
\hline CD at $5 \%$ & 2.208 & 2.111 & 2.034 & N.S & 0.782 & 0.631 & N.S & N.S & N.S & N.S & N.S & N.S \\
\hline \multicolumn{13}{|l|}{ Bio-mulches } \\
\hline $\mathbf{M}_{1}$ & 63.92 & 64.46 & 64.19 & 17.50 & 18.23 & 17.86 & 2.82 & 2.73 & 2.77 & 3.82 & 3.73 & 3.78 \\
\hline $\mathbf{M}_{2}$ & 64.54 & 64.46 & 64.50 & 19.63 & 19.97 & 19.80 & 2.92 & 2.69 & 2.81 & 3.73 & 3.80 & 3.77 \\
\hline $\mathbf{M}_{3}$ & 62.71 & 63.21 & 62.96 & 17.39 & 17.91 & 17.65 & 2.57 & 2.51 & 2.54 & 4.04 & 4.00 & 4.02 \\
\hline $\mathbf{M}_{4}$ & 67.34 & 67.78 & 67.56 & 19.72 & 19.51 & 19.61 & 2.97 & 2.91 & 2.94 & 3.82 & 3.81 & 3.82 \\
\hline CD at $5 \%$ & 2.208 & 2.11 & 4.064 & N.S & 0.782 & 0.631 & N.S & N.S & N.S & N.S & N.S & N.S \\
\hline \multicolumn{13}{|l|}{ Interactions } \\
\hline $\mathbf{N}_{1} \mathbf{M}_{1}$ & 61.81 & 63.72 & 62.77 & 18.20 & 17.21 & 17.71 & 2.78 & 2.56 & 2.67 & 4.00 & 3.89 & 3.95 \\
\hline $\mathbf{N}_{1} \mathbf{M}_{2}$ & 62.49 & 63.81 & 63.15 & 21.94 & 19.99 & 20.97 & 2.89 & 2.44 & 2.66 & 3.78 & 4.00 & 3.89 \\
\hline $\mathbf{N}_{1} \mathbf{M}_{3}$ & 60.01 & 61.96 & 60.99 & 16.28 & 18.78 & 17.53 & 2.56 & 2.22 & 2.39 & 4.40 & 4.13 & 4.27 \\
\hline $\mathbf{N}_{1} \mathbf{M}_{4}$ & 68.60 & 67.98 & 68.29 & 17.84 & 18.54 & 18.19 & 2.78 & 2.78 & 2.78 & 3.84 & 3.94 & 3.89 \\
\hline $\mathbf{N}_{2} \mathbf{M}_{1}$ & 62.03 & 61.97 & 62.00 & 15.79 & 18.68 & 17.24 & 2.94 & 2.67 & 2.80 & 3.56 & 3.73 & 3.65 \\
\hline $\mathbf{N}_{2} \mathbf{M}_{2}$ & 68.61 & 65.29 & 66.95 & 17.82 & 20.50 & 19.16 & 2.94 & 2.78 & 2.86 & 3.78 & 3.96 & 3.87 \\
\hline $\mathbf{N}_{2} \mathbf{M}_{3}$ & 61.98 & 62.98 & 62.48 & 17.66 & 17.03 & 17.35 & 2.67 & 2.56 & 2.61 & 3.67 & 4.02 & 3.85 \\
\hline $\mathbf{N}_{2} \mathbf{M}_{4}$ & 65.10 & 66.77 & 65.94 & 20.53 & 19.48 & 20.01 & 3.33 & 3.22 & 3.27 & 3.59 & 3.98 & 3.79 \\
\hline $\mathbf{N}_{3} \mathbf{M}_{1}$ & 66.88 & 67.52 & 67.20 & 16.68 & 17.20 & 16.94 & 2.66 & 2.81 & 2.73 & 4.11 & 3.86 & 3.99 \\
\hline $\mathbf{N}_{3} \mathbf{M}_{2}$ & 62.04 & 61.68 & 61.86 & 18.72 & 17.95 & 18.34 & 3.00 & 2.78 & 2.89 & 3.96 & 3.67 & 3.82 \\
\hline $\mathbf{N}_{3} \mathbf{M}_{3}$ & 65.91 & 65.57 & 65.74 & 17.10 & 17.51 & 17.31 & 2.44 & 2.67 & 2.55 & 4.22 & 4.00 & 4.11 \\
\hline $\mathbf{N}_{3} \mathbf{M}_{4}$ & 66.24 & 65.90 & 66.07 & 19.43 & 18.14 & 18.79 & 2.67 & 2.56 & 2.61 & 4.56 & 4.11 & 4.34 \\
\hline $\mathbf{N}_{4} \mathbf{M}_{1}$ & 64.97 & 64.63 & 64.80 & 19.31 & 19.84 & 19.57 & 2.89 & 2.89 & 2.89 & 3.62 & 3.44 & 3.53 \\
\hline $\mathbf{N}_{4} \mathbf{M}_{2}$ & 65.00 & 67.06 & 66.03 & 20.03 & 21.44 & 20.74 & 2.86 & 2.78 & 2.82 & 3.42 & 3.59 & 3.51 \\
\hline $\mathbf{N}_{4} \mathbf{M}_{3}$ & 62.93 & 62.32 & 62.63 & 18.51 & 18.32 & 18.42 & 2.60 & 2.60 & 2.60 & 3.89 & 3.86 & 3.88 \\
\hline $\mathbf{N}_{4} \mathbf{M}_{4}$ & 69.40 & 70.45 & 69.93 & 21.07 & 21.87 & 21.47 & 3.11 & 3.10 & 3.10 & 3.31 & 3.22 & 3.27 \\
\hline $\mathrm{CD}$ at $5 \%$ & 4.416 & 4.22 & 4.064 & 2.102 & 1.565 & 1.785 & N.S & N.S & N.S & N.S & N.S & N.S \\
\hline
\end{tabular}


Table.2 Effect of organic nutrients and bio-mulches on growth parameters of okra

\begin{tabular}{|c|c|c|c|c|c|c|c|c|c|}
\hline Treatment* & $\begin{array}{l}\text { Days to } \\
50 \% \\
\text { flowering }\end{array}$ & & & $\begin{array}{l}\text { Days to } \\
\text { first } \\
\text { harvest }\end{array}$ & & & $\begin{array}{l}\text { Days to } \\
\text { last } \\
\text { harvest }\end{array}$ & & \\
\hline & 2017 & 2018 & pooled & 2017 & 2018 & pooled & 2017 & 2018 & pooled \\
\hline \multicolumn{10}{|c|}{ Nutrient sources } \\
\hline $\mathbf{N}_{1}$ & 49.31 & 49.87 & 49.59 & 56.50 & 56.43 & 56.47 & 82.66 & 80.66 & 81.66 \\
\hline $\mathbf{N}_{2}$ & 49.57 & 50.37 & 49.97 & 56.49 & 56.96 & 56.73 & 85.73 & 84.18 & 84.95 \\
\hline $\mathbf{N}_{3}$ & 50.15 & 49.64 & 49.89 & 57.84 & 57.68 & 57.76 & 83.73 & 80.70 & 82.21 \\
\hline $\mathbf{N}_{4}$ & 48.19 & 48.18 & 48.18 & 54.19 & 56.20 & 55.19 & 86.20 & 86.28 & 86.24 \\
\hline CD at $5 \%$ & 1.232 & 0.793 & 0.713 & 1.039 & 1.078 & 0.717 & 0.686 & 0.663 & 0.462 \\
\hline \multicolumn{10}{|l|}{ Bio-mulches } \\
\hline $\mathbf{M}_{1}$ & 48.69 & 49.42 & 49.05 & 56.38 & 56.16 & 56.27 & 84.34 & 84.32 & 84.33 \\
\hline $\mathbf{M}_{2}$ & 48.69 & 48.88 & 48.79 & 55.10 & 56.55 & .82 & 84.81 & 84.24 & 84.52 \\
\hline $\mathbf{M}_{3}$ & 51.05 & 50.14 & 50.60 & 58.01 & 58.10 & 58.06 & 81.96 & 79.01 & 80.49 \\
\hline $\mathbf{M}_{4}$ & 48.78 & 49.61 & 49.19 & 55.54 & 56.46 & 56.00 & 87.21 & 84.26 & 85.73 \\
\hline CD at $5 \%$ & N.S & N.S & N.S & 1.039 & 1.078 & 0.717 & 0.686 & 0.663 & 0.462 \\
\hline \multicolumn{10}{|l|}{ Interactions } \\
\hline $\mathbf{N}_{1} \mathbf{M}_{1}$ & 48.50 & 48.30 & 48.40 & 55.93 & 54.23 & 55.08 & 81.87 & 82.60 & 82.23 \\
\hline $\mathbf{N}_{1} \mathbf{M}_{2}$ & 49.50 & 49.53 & 49.52 & 56.83 & 56.20 & 56.52 & 84.03 & 83.10 & 83.56 \\
\hline $\mathbf{N}_{1} \mathbf{M}_{3}$ & 50.00 & 51.53 & 50.77 & 58.67 & 58.87 & 58.77 & 80.43 & 76.43 & 78.43 \\
\hline $\mathbf{N}_{1} \mathbf{M}_{4}$ & 49.23 & 50.10 & 49.67 & 54.57 & 56.43 & 55.50 & 84.30 & 80.53 & 82.41 \\
\hline $\mathbf{N}_{2} \mathbf{M}_{1}$ & 48.43 & 49.37 & 48.90 & 56.80 & 56.42 & 56.61 & 86.43 & 85.80 & 86.11 \\
\hline $\mathbf{N}_{2} \mathbf{M}_{2}$ & 48.80 & 49.30 & 49.05 & 53.80 & 56.30 & 55.05 & 86.17 & 84.77 & 85.47 \\
\hline $\mathbf{N}_{2} \mathbf{M}_{3}$ & 51.90 & 52.40 & 52.15 & 58.90 & 58.40 & 58.65 & 81.20 & 80.37 & 80.78 \\
\hline $\mathbf{N}_{2} \mathbf{M}_{4}$ & 49.13 & 50.40 & 49.77 & 56.47 & 56.73 & 56.60 & 89.13 & 85.80 & 87.46 \\
\hline $\mathbf{N}_{3} \mathbf{M}_{1}$ & 49.17 & 50.46 & 49.82 & 57.10 & 57.47 & 57.29 & 85.40 & 84.40 & 84.90 \\
\hline $\mathbf{N}_{3} \mathbf{M}_{2}$ & 49.38 & 49.27 & 49.33 & 56.33 & 56.30 & 56.32 & 84.70 & 80.53 & 82.61 \\
\hline $\mathbf{N}_{3} \mathbf{M}_{3}$ & 51.67 & 48.48 & 50.08 & 59.60 & 59.53 & 59.57 & 80.20 & 76.70 & 78.45 \\
\hline $\mathbf{N}_{3} \mathbf{M}_{4}$ & 50.38 & 50.33 & 50.36 & 58.33 & 57.40 & 57.87 & 84.63 & 81.17 & 82.90 \\
\hline $\mathbf{N}_{4} \mathbf{M}_{1}$ & 48.67 & 49.53 & 49.10 & 55.70 & 56.50 & 56.10 & 83.67 & 84.47 & 84.07 \\
\hline $\mathbf{N}_{4} \mathbf{M}_{2}$ & 47.08 & 47.43 & 47.26 & 53.43 & 57.40 & 55.42 & 84.33 & 88.57 & 86.45 \\
\hline $\mathbf{N}_{4} \mathbf{M}_{3}$ & 50.62 & 48.16 & 49.39 & 54.87 & 55.62 & 55.24 & 86.03 & 82.57 & 84.30 \\
\hline $\mathbf{N}_{4} \mathbf{M}_{4}$ & 46.39 & 47.59 & 46.99 & 52.77 & 55.27 & 54.02 & 90.77 & 89.53 & 90.15 \\
\hline CD at $5 \%$ & 2.463 & 1.585 & N.S & 2.077 & 2.155 & 2.028 & 1.371 & 1.326 & 1.307 \\
\hline
\end{tabular}


Table.3 Effect of organic nutrients and bio-mulches physiological parameters of okra

\begin{tabular}{|c|c|c|c|c|c|c|c|c|c|}
\hline Treatment* & $\begin{array}{l}\text { Leaf chlorophyll } \\
\text { content at } 60 \text { DAS } \\
\text { (SPAD valuer) }\end{array}$ & & & $\begin{array}{l}\text { Crop growth rate } \\
\text { at } 45-60 \text { DAS } \\
\left(\text { gram } / \mathbf{m}^{2} / \text { day }\right)\end{array}$ & & & $\begin{array}{l}\text { Dry matter } \\
\text { accumulation at } \\
60 \text { DAS }(\%)\end{array}$ & & \\
\hline & 2017 & 2018 & Pooled & 2017 & 2018 & Pooled & 2017 & 2018 & pooled \\
\hline \multicolumn{10}{|l|}{ Nutrient sources } \\
\hline $\mathbf{N}_{1}$ & 32.30 & 33.90 & 33.10 & 3.84 & 3.84 & 3.84 & 18.41 & 19.03 & 18.72 \\
\hline $\mathbf{N}_{2}$ & 37.81 & 38.83 & 38.32 & 4.25 & 4.27 & 4.26 & 18.69 & 18.95 & 18.82 \\
\hline $\mathbf{N}_{3}$ & 30.58 & 31.48 & 31.03 & 3.66 & 3.84 & 3.75 & 16.99 & 17.61 & 17.30 \\
\hline $\mathbf{N}_{4}$ & 39.02 & 40.75 & 39.88 & 4.61 & 4.58 & 4.59 & 24.07 & 23.64 & 23.85 \\
\hline CD at $5 \%$ & 2.392 & 1.176 & 1.318 & 0.371 & 0.264 & 0.221 & 2.044 & 2.094 & 1.458 \\
\hline \multicolumn{10}{|l|}{ Bio-mulches } \\
\hline $\mathbf{M}_{1}$ & 34.90 & 37.14 & 36.02 & 4.30 & 4.29 & 4.29 & 19.39 & 19.51 & 19.45 \\
\hline $\mathbf{M}_{2}$ & 36.10 & 36.16 & 36.13 & 4.09 & 4.13 & 4.11 & 19.99 & 19.81 & 19.90 \\
\hline $\mathbf{M}_{3}$ & 32.17 & 33.68 & 32.93 & 3.32 & 3.49 & 3.41 & 17.06 & 18.14 & 17.60 \\
\hline $\mathbf{M}_{4}$ & 36.54 & 37.97 & 37.25 & 4.65 & 4.63 & 4.64 & 21.70 & 21.78 & 21.74 \\
\hline CD at $5 \%$ & 2.392 & 1.176 & 1.318 & 0.371 & 0.264 & 0.221 & 2.044 & 2.094 & 1.458 \\
\hline \multicolumn{10}{|l|}{ Interactions } \\
\hline $\mathbf{N}_{1} \mathbf{M}_{1}$ & 32.82 & 34.14 & 33.48 & 3.96 & 3.89 & 3.93 & 16.23 & 16.89 & 16.56 \\
\hline $\mathbf{N}_{1} \mathbf{M}_{2}$ & 32.13 & 33.81 & 32.97 & 4.12 & 4.09 & 4.11 & 18.60 & 19.13 & 18.87 \\
\hline $\mathbf{N}_{1} \mathbf{M}_{3}$ & 30.03 & 31.84 & 30.94 & 3.12 & 3.21 & 3.17 & 19.20 & 19.78 & 19.49 \\
\hline $\mathbf{N}_{1} \mathbf{M}_{4}$ & 34.20 & 35.81 & 35.01 & 4.16 & 4.18 & 4.17 & 19.59 & 20.32 & 19.96 \\
\hline $\mathbf{N}_{2} \mathrm{M}_{1}$ & 37.78 & 40.91 & 39.35 & 4.31 & 4.34 & 4.33 & 18.01 & 18.23 & 18.12 \\
\hline $\mathbf{N}_{2} \mathbf{M}_{2}$ & 40.97 & 39.13 & 40.05 & 4.27 & 4.29 & 4.28 & 20.51 & 20.21 & 20.36 \\
\hline $\mathbf{N}_{2} \mathbf{M}_{3}$ & 34.17 & 35.10 & 34.64 & 3.53 & 3.66 & 3.60 & 17.19 & 18.01 & 17.60 \\
\hline $\mathbf{N}_{2} \mathbf{M}_{4}$ & 38.33 & 40.16 & 39.25 & 4.87 & 4.78 & 4.83 & 19.03 & 19.34 & 19.19 \\
\hline $\mathbf{N}_{3} \mathbf{M}_{1}$ & 30.33 & 32.13 & 31.23 & 4.12 & 4.14 & 4.13 & 17.23 & 17.14 & 17.19 \\
\hline $\mathbf{N}_{3} \mathbf{M}_{2}$ & 31.51 & 31.38 & 31.45 & 3.40 & 3.69 & 3.55 & 17.85 & 18.13 & 17.99 \\
\hline $\mathbf{N}_{3} \mathbf{M}_{3}$ & 28.21 & 28.94 & 28.58 & 2.79 & 3.12 & 2.96 & 14.33 & 16.73 & 15.53 \\
\hline $\mathbf{N}_{3} \mathbf{M}_{4}$ & 32.26 & 33.45 & 32.86 & 4.34 & 4.41 & 4.38 & 18.55 & 18.43 & 18.49 \\
\hline $\mathbf{N}_{4} \mathbf{M}_{1}$ & 38.67 & 41.39 & 40.03 & 4.81 & 4.78 & 4.80 & 26.10 & 25.76 & 25.93 \\
\hline $\mathbf{N}_{4} \mathbf{M}_{2}$ & 39.77 & 40.31 & 40.04 & 4.57 & 4.43 & 4.50 & 22.99 & 21.78 & 22.39 \\
\hline $\mathbf{N}_{4} \mathbf{M}_{3}$ & 36.26 & 38.85 & 37.56 & 3.85 & 3.96 & 3.91 & 17.53 & 18.02 & 17.78 \\
\hline $\mathbf{N}_{4} \mathbf{M}_{4}$ & 41.36 & 42.46 & 41.91 & 5.21 & 5.13 & 5.17 & 29.64 & 29.01 & 29.33 \\
\hline CD at $5 \%$ & 4.784 & 2.353 & 3.728 & 0.742 & 0.528 & 0.442 & 4.088 & 4.189 & 2.916 \\
\hline
\end{tabular}


Table.4 Effect of organic nutrients and bio-mulches on yield parameters of okra

\begin{tabular}{|c|c|c|c|c|c|c|c|c|c|}
\hline Treatment* & $\begin{array}{l}\text { No. of fruit } \\
\text { per plant }\end{array}$ & & & $\begin{array}{l}\text { Yield per } \\
\text { plant } \\
\text { (gram) }\end{array}$ & & & $\begin{array}{l}\text { Total } \\
\text { yield per } \\
\text { ha (ton) }\end{array}$ & & \\
\hline & 2017 & 2018 & Pooled & 2017 & 2018 & Pooled & 2017 & 2018 & Pooled \\
\hline \multicolumn{10}{|c|}{ Nutrient sources } \\
\hline $\mathbf{N}_{1}$ & 17.86 & 18.13 & 17.99 & 305.03 & 300.37 & 302.70 & 12.97 & 13.62 & 13.30 \\
\hline $\mathbf{N}_{2}$ & 18.99 & 19.21 & 19.10 & 315.92 & 320.50 & 318.21 & 13.74 & 14.04 & 13.90 \\
\hline $\mathbf{N}_{3}$ & 16.79 & 18.28 & 17.53 & 288.92 & 280.32 & 284.62 & 12.87 & 12.96 & 12.92 \\
\hline $\mathbf{N}_{4}$ & 19.40 & 20.49 & 19.94 & 319.41 & 324.90 & 322.15 & 13.99 & 14.80 & 14.39 \\
\hline CD at $5 \%$ & 0.719 & 0.741 & 0.498 & 5.206 & 5.532 & 3.826 & 2.447 & 2.308 & 1.644 \\
\hline \multicolumn{10}{|l|}{ Bio-mulches } \\
\hline $\mathbf{M}_{1}$ & 18.25 & 17.99 & 18.12 & 308.27 & 305.66 & 306.96 & 13.61 & 13.56 & 13.59 \\
\hline $\mathbf{M}_{2}$ & 18.65 & 19.32 & 18.99 & 310.06 & 298.36 & 304.21 & 13.89 & 13.75 & 13.82 \\
\hline $\mathbf{M}_{3}$ & 15.87 & 17.49 & 16.68 & 281.95 & 301.37 & 291.66 & 11.95 & 12.64 & 12.30 \\
\hline $\mathbf{M}_{4}$ & 20.27 & 21.30 & 20.79 & 329.01 & 320.70 & 324.86 & 14.12 & 15.46 & 14.79 \\
\hline CD at $5 \%$ & 0.719 & 0.741 & 0.498 & 5.206 & 5.532 & 3.826 & 2.447 & 2.308 & 1.644 \\
\hline \multicolumn{10}{|l|}{ Interactions } \\
\hline $\mathbf{N}_{1} \mathbf{M}_{1}$ & 16.78 & 16.67 & 16.73 & 304.05 & 301.47 & 302.76 & 13.30 & 13.41 & 13.35 \\
\hline $\mathbf{N}_{1} \mathbf{M}_{2}$ & 18.22 & 18.17 & 18.20 & 310.41 & 277.47 & 293.94 & 13.80 & 13.89 & 13.84 \\
\hline $\mathbf{N}_{1} \mathbf{M}_{3}$ & 16.87 & 17.56 & 17.22 & 281.27 & 309.14 & 295.20 & 11.61 & 12.73 & 12.17 \\
\hline $\mathbf{N}_{1} \mathbf{M}_{4}$ & 19.56 & 20.11 & 19.84 & 324.40 & 313.40 & 318.90 & 13.18 & 14.46 & 13.82 \\
\hline $\mathbf{N}_{2} \mathbf{M}_{1}$ & 19.82 & 18.00 & 18.91 & 318.78 & 312.10 & 315.44 & 13.79 & 13.74 & 13.76 \\
\hline $\mathbf{N}_{2} \mathbf{M}_{2}$ & 20.39 & 20.15 & 20.27 & 321.85 & 316.29 & 319.07 & 14.23 & 13.38 & 13.80 \\
\hline $\mathbf{N}_{2} \mathbf{M}_{3}$ & 15.61 & 17.48 & 16.55 & 289.18 & 322.43 & 305.80 & 12.31 & 12.05 & 12.18 \\
\hline $\mathbf{N}_{2} \mathbf{M}_{4}$ & 20.15 & 21.20 & 20.68 & 333.86 & 331.19 & 332.52 & 14.66 & 17.01 & 15.83 \\
\hline $\mathbf{N}_{3} \mathbf{M}_{1}$ & 17.78 & 17.82 & 17.80 & 290.93 & 281.27 & 286.10 & 13.53 & 13.01 & 13.27 \\
\hline $\mathbf{N}_{3} \mathbf{M}_{2}$ & 15.78 & 18.41 & 17.10 & 292.41 & 277.93 & 286.07 & 13.41 & 13.43 & 13.42 \\
\hline $\mathbf{N}_{3} \mathbf{M}_{3}$ & 14.48 & 16.48 & 15.48 & 264.10 & 268.68 & 266.39 & 11.77 & 12.46 & 12.11 \\
\hline $\mathbf{N}_{3} \mathbf{M}_{4}$ & 19.11 & 20.39 & 19.75 & 306.45 & 293.40 & 299.92 & 12.78 & 12.95 & 12.86 \\
\hline $\mathbf{N}_{4} \mathbf{M}_{1}$ & 18.60 & 19.47 & 19.04 & 319.32 & 327.79 & 323.55 & 13.81 & 14.10 & 13.95 \\
\hline $\mathbf{N}_{4} \mathbf{M}_{2}$ & 20.22 & 20.55 & 20.39 & 313.76 & 321.74 & 317.75 & 14.13 & 14.32 & 14.22 \\
\hline $\mathbf{N}_{4} \mathbf{M}_{3}$ & 16.50 & 18.45 & 17.48 & 293.24 & 305.23 & 299.23 & 12.13 & 13.34 & 12.73 \\
\hline $\mathbf{N}_{4} \mathbf{M}_{4}$ & 22.26 & 23.50 & 22.88 & 351.34 & 344.83 & 348.08 & 15.88 & 17.43 & 16.65 \\
\hline CD at $5 \%$ & 1.437 & 1.483 & 0.996 & 10.411 & 11.064 & 7.652 & 4.894 & 4.615 & 3.288 \\
\hline
\end{tabular}

*Treatment details are in materials and methods 


\section{Effect of bio mulches}

The pooled data presented in tables 1-4 revealed that the vegetable legume plant residue mulch $\left(\mathrm{M}_{4}\right)$ emerged as superior among other types of bio mulches in regarding to growth, physiology and yield of okra plant. The bio mulch $\mathrm{M}_{4}$ resulted highest plant height at 60 DAS $(67.56 \mathrm{~cm})$, stem girth at the time of last harvesting $(19.61 \mathrm{~mm})$, days to last harvest (85.73 days). The dry water hyacinth mulch $\left(\mathrm{M}_{2}\right)$ took minimum days to $50 \%$ flowering (48.79 days) and days to first harvest (55.82 days) in pooled but $\mathrm{M}_{4}$ resulted maximum days to last harvest $(85.73$ days). In relation to physiological parameter $\mathrm{M}_{4}$ exhibited highest chlorophyll content (37.25), crop growth rate at 45-60 DAS (4.64 $\mathrm{gram} / \mathrm{m}^{2} /$ day) and maximum dry matter at 60 DAS $(21.74 \%)$. The maximum no. of fruit per plant (20.79), yield per plant (324.86 gram/ plant) and total yield (14.79 t/ha) were also observed by $\mathrm{M}_{4}$.

The application of vegetable legume plant residue mulch having low $\mathrm{C}: \mathrm{N}$ ratio than other bio mulches may be increased soil micro flora and provide major plant nutrient like nitrogen for better crop growth which subsequently triggered crop growth , metabolism and yield.

\section{Effect of interaction of organic nutrient and bio mulches}

The interaction effect of organic nutrient sources and bio mulches showed marked influence on different growth, physiology and yield attributes of okra plant. The pooled results revealed that maximum plant height at 60 DAS $(69.93 \mathrm{~cm})$, lower days to $50 \%$ flowering (46.99 days), earlier harvesting (54.02 days) were obtained when okra was grown under the treatment combination comprising of Azophos biofertiliser enriched vermicompost $(5 t / h a)$ with three times foliar application of seaweed extract (at 30 DAS, 40 DAS, 50 DAS) along with vegetable legume plant residue as mulch material $\left(\mathrm{N}_{4} \mathrm{M}_{4}\right)$. The treatment combination $\mathrm{N}_{4} \mathrm{M}_{4}$ resulted maximum leaf chlorophyll content (41.91 SPAD value) and crop growth rate (5.17 gram $/ \mathrm{m}^{2} /$ day). The same treatment combination also recorded maximum number of fruits per plant (22.88), highest total yield per plant (348.08 gram), total yield per hectare (16.65 t/ hectare).

The findings indicated that combined application of vermi compost with biofertiliser along with foliar application of sea weed extract have pronounced effect on yield attributes. This might be properties of vermin compost and bio fertilizer that enhanced soil physicochemical properties by solubilizing and mineralizing the reserve nutrient content of soil. Further the foliar application of seaweed extract act as a plant soil conditioner by increasing the plant metabolism and plant physiology (Fan et al., 2013).

The experiment concluded that Azophos biofertiliser enriched vermicompost with three time foliar spray of sea weed extract ( at 30 DAS, 40 DAS and 50 DAS) along with dry vegetable legume plant residue as mulch material emerged as best treatment combination with respect to every attribute for organic okra cultivation under Terai Zone Of west Bengal.

\section{References}

Adams, C.F., 1975. Nutritive value of American foods in common units, U.S. Department of Agriculture, Agriculture Handbook, 425: 29.

Aydin, A., Kant, C and Turan, M., 2012. Humic acid application alleviates salinity stress of bean (Phaseolus vulgaris L.) plants decreasing 
membrane leakage. African Journal of Agriculture Research, 7: 1073-1086.

Brown, J.E., and Channel-Butcher C., 2001. Black plastic mulch and drip irrigation affect growth and performance of bell pepper, Journal of Vegetable Crop Production, 7 (2): 109 - 112.

Dhankhar, S.K., and Mishra, J.P., 2005. Objectives of okra breeding, Journal of New Seeds, 6(2):195-209

Fan, D., Hodges, D.M., Critchley, A.T. and Prithiviraj B., 2013. A commercial extract of brown macroalga (Ascophyllum nodosum) affects yield and nutritional quality of spinach in vitro. Communications in Soil Science and Plant Analysis, 44:1873-1884.

Gopinathan, R and Prakash, M,. 2014. Effect of vermicompost enriched with biofertilizers on the productivity of tomato (Lycopersicum esculentum mill.). International Journal of Current Microbiology and Applied Sciences, 3 (9):1238-1245

Hochmuth, G., 1998. What to do with all that mulch?, American Vegetable Grower. $46(4): 45$.

Kumari, R., Kaur, I and Bhatnagar, A.K., 2011. Effect of aqueous extract of Sargassum johnstonii Setchell \& Gardner on growth, yield and quality of Lycopersicon esculentum Mill., Journal of Applied Phycology, 22(3): 623-633

Lamont, W J Jr., 1993. Plastic mulches for the production of vegetable crops, Horticulture Technology, 3 (1): 35 - 39. Nandkarni, K. M., 1927. Vegetable crops. Advances in Horticulture (Chadha K. Land Kalloo. G. eds.). Malhotra Publishing House, New Delhi, 5:105129.

Rice, P.J., McConnell L.L., Heighton L.P., Sadeghi, A.M., Isensee, A.L., Teasdale, J.R., Abdul- Baki, A.A., HarmenFetcho, J.A. and Hapeman, C.J., 2001. Run off loss of pesticides and Soil: A comparison between vegetative mulch and plastic mulch in vegetable production systems, Journal of Environmental Quality, 30 (5): 1808 1821.

Yawalkar, K.S., 1965. Bhindi or Ladies Finger, Vegetable Crops of India $\left(3^{\text {rd }}\right.$ Eds.), Agri-Horticultural Publishing House, Cornell University, 66

Zodape, S.T., Gupta, A., Bhandari, S.C., Rawat, U. S., Chaudhry, D. R., Eswaran, K and Chikara J., 2011. Foliar application of seaweed sap as biostimulant for enhancement of yield and quality of tomato, Journal of Scientific and Industrial Research, 70: 215-219.

Zodape, S.T., Kawarkhe, V.J., Patolia, J.S., and Warade, A.D., 2008. Effect of liquid seaweed fertilizer on yield and quality of okra. Journal of scientific and Industrial Research, 67 (12):1115-1117.

\section{How to cite this article:}

Subhalaxmi Mishra, Satyamaya Satapathy, Ranjit Chatterjee and Koushik Saha. 2019. Effect of Organic Nutrients and Bio-mulches on Growth, Physiology and Yield of Okra (Abelmoschus esculentus L. Moench). Int.J.Curr.Microbiol.App.Sci. 8(09): 204-212. doi: https://doi.org/10.20546/ijcmas.2019.809.026 